\title{
SELETIVIDADE DE INSETICIDAS A Neoseiulus californicus MCGREGOR (ACARI: PHYTOSEIIDAE) EM MACIEIRA, NO RIO GRANDE DO SUL ${ }^{1}$
}

\author{
LINO BITTENCOURT MONTEIRO²
}

\begin{abstract}
RESUMO - Testes para determinar os efeitos de agroquímicos homologados para macieira foram realizados em laboratório sobre Neoseiulus californicus (Acari: Phytoseiidae). Os indivíduos testados foram coletados de um pomar comercial da Agriflor Ltda, em Vacaria, Rio Grande do Sul, após várias liberações inoculativas. Os inseticidas utilizados foram os tradicionalmente recomendados para o controle de pragas, principalmente mosca-das-frutas Anastrepha fraterculus (Diptera: Tephritidae). A oviposição e a mortalidade dos ácaros foram avaliadas 12; 24; 48 e 96 horas após a pulverização, cujos produtos foram classificados em quatro classes de toxicidade (IOBC/WPRS). Azinphos ethyl, deltametrina e fenthion provocaram 100\% de mortalidade, sendo que dimethoate, fenitrotion, paration, phosmet e triclorfon foram levemente nocivos (classe 2). Malation foi considerado neutro para esta população.
\end{abstract}

Termos para indexação: fitoseídeos, macieira, ácaro vermelho, controle biológico, seletividade

\section{AGROCHEMICAL SELECTIVITY TO Neoseiulus californicus MCGREGOR (ACARI: PHYTOSEIIDAE) ON APPLE IN RIO GRANDE DO SUL.}

\begin{abstract}
The side-effects of agrochemical to Neoseiulus californicus (Acari: Phytoseiidae) were studied in laboratory. The mites were collected in commercial apple orchard of Agropastoril Rincão das Flores, in Vacaria, Rio Grande do Sul, Brazil, after successive inoculative releases. The insecticids used were recommended to control of same pest, as Anastrepha fraterculus (Diptera: Tephritidae). The reproduction effect and mortality were evalued 12, 24, 48 and 96 hours after pulverization, while the agrochemical were ranked in toxicity classes, according to IOBC/WPRS. Azinphos ethyl, deltametrina e fenthion were harmful with ca. $100 \%$ of mortality in 24 hours, dimethoate, fenitrotion, paration, phosmet e triclorfon were slightly harmful (class 2). Malation was harmless.
\end{abstract}

Index terms: fitoseid, apple orchard, European red mites, biological control, selectivity

\section{INTRODUÇÃO}

Entre os ácaros predadores, os pertencentes à família Phytoseiidae são os mais estudados e utilizados em programas de controle biológico (McMurtry et al., 1970; Fauvel \& Atger, 1981). Neoseiulus californicus (McGregor) (Acari: Phytoseiidae) é um ácaro predador que ocorre no Sul da Europa e nos Países Andinos (Moraes et al., 1986).

Em 1992, este ácaro predador foi introduzido em um pomar de macieira da Agropastoril Rincão das Flores, em Vacaria, Rio Grande do Sul, para o controle do ácaro vermelho Panonychus ulmi Koch (Acari: Tetranychidae). A sobrevivência dos inimigos naturais depende do programa fitossanitário empregado nos pomares. Na região de Vacaria, ocorre a presença de várias pragas, entre as quais se destaca a mosca-das-frutas Anastrepha fraterculus (Wied) (Diptera: Tephritidae), que é a espécie dominante na região, com freqüência de $96 \%$ das capturas em caça-moscas (Salles \& Kovaleski, 1990a). Muitos dos agroquímicos recomendados (Bleicher, 1982; Salles \& Kovaleski, 1990b; Salles, 1991) são extremamente tóxicos para ácaros predadores (Besson et al., 1978; SPV, 1983; ANPP 1987). A presença de outras pragas, como Grapholita molesta (Busck.) (Lepidoptera: Tortricidae) e Naupactus sp. (Coleoptera: Curculionidae), cujos conhecimentos bioecológicos na região não foram devidamente aprofundados, conduzem a tratamentos preventivos.

O objetivo deste trabalho foi conhecer os efeitos provocados pelos produtos fitossanitários sobre o ácaro predador $N$. californicus utilizados no controle de pragas em macieira.

\section{MATERIAL E MÉTODOS}

Obtenção do ácaro predador. $N$. californicus foi obtido do Centro Nacional de Pesquisa do Meio Ambiente (CNPMA), unidade da Empresa Brasileira de Pesquisa Agropecuária (EMBRAPA), em Jaguariúna-SP, e introduzido no pomar de estudo, em outubro de 1992 (Monteiro, 1994). Aproximadamente 500000 ácaros predadores foram liberados em uma área de 2 ha, os quais foram coletados 6 meses após sua liberação. A população utilizada para o tratamento-testemunha foi originada da criação de rotina, não sofrendo nenhum tratamento químico.

Condução experimental. Os ácaros predadores utilizados para estes testes foram previamente multiplicados a fim de obter uma população de idade conhecida. Para tanto, seis fêmeas de $N$. californicus foram colocadas em uma arena de plástico preto $(2 \times 2 \mathrm{~cm})$, totalizando um conjunto de 12 arenas por tratamento, depositadas sobre algodão úmido, que, por sua vez, está em uma

1 (Trabalho 042/2001). Recebido: 16/02/2001. Aceito para publicação: 04/10/2001.

2 Eng $^{\circ}$ Agrônomo, D. Sc., Professor do DFF/UFPR, rua dos Funcionários, 1540, 80035-050, Curitiba, PR, lbmonteiro@mais.sul.com.br 
caixa de polietileno $(13 \times 7 \mathrm{~cm})$, semelhante à utilizada em estudos de biologia por Monteiro (1991). Para estimular a ovoposição, foi colocada nas arenas mechas de algodão cobertas por uma lamínula de microscopia (18 x 18 mm) (Overmeer, 1985). Após 5 horas ovopositando, as fêmeas foram retiradas das células, cujos ovos, larvas e ninfas foram observados até a obtenção de fêmeas adultas de mesma idade, alimentadas com ácaro rajado Tetranychus urticae. Destas, duas fêmeas com dois dias de emergência e acasaladas foram colocadas em cada arena, as quais foram previamente pulverizadas com os inseticidas e submetidas à secagem natural por uma hora. Os tratamentos foram realizados com um atomizador marca Desaga ${ }^{\circ} 124000$, Heidelberg, durante 20 segundos por arena e produzindo uma calda de $1,0 \pm 0,25 \mathrm{ml}$ de calda por $\mathrm{cm}^{2}$. Durante o período de avaliação, os ácaros foram alimentados com pólen de mamoneira (Ricinus communis) depositado sobre uma lamínula de microscopia, o que impediu o contado com os inseticidas. Utilizaram-se 12 repetições (arenas), totalizando 24 fêmeas para cada tratamento. Os ingredientes ativos testados encontram-se na Tabela 1, os quais foram recomendados para o controle de A. fraterculus (Bleicher, 1982; Salles \& Kovaleski, 1990b; Salles, 1991), pulgões e lagartas (Andrei, 1990). A mortalidade e oviposição das fêmeas foram observadas a 12; 24; 72 e 96 horas após a pulverização; os intervalos de tempo para as avaliações foram escolhidos a fim de identificar os inseticidas que apresentam efeito de choque sobre os ácaros predadores; entretanto, os resultados finais apresentados neste estudo representam os dados obtidos após 96 horas da pulverização. Entre a introdução do ácaro predador no pomar e a coleta dos mesmos para os testes, foram utilizados os seguintes inseticidas: malathion (3/11 e 17/11/92), phosmet (1-12, 14-12-92 e 14-1-93), fenthion (7-12 e 21-12-92), triclorfon (14-1-93) e sumithion (28-1 e 9-2-93).

Critérios de avaliação. O efeito negativo (E) foi calculado pelas indicações da IOBC/WPRS (International Organization for Biological and Integrated Control of Noxious Animal and Plants/ West Paleartic Regional Section) (Hassan et al., 1985; Bakker et al., 1992), sendo $\mathrm{E}=100 \%-\left(100 \%-\mathrm{M}_{\mathrm{c}}\right) \times \mathrm{E}_{\mathrm{r}}$, onde $\mathrm{M}_{\mathrm{c}}=$ mortalidade corrigida (Abbott, 1925) e $\mathrm{E}_{\mathrm{r}}=$ efeito na reprodução. $\mathrm{E}_{\mathrm{r}}=\mathrm{R}_{\text {tratamento }}$ ' $\mathrm{R}_{\text {testemunha, }}$, onde $\mathrm{R}$ é o número de ovos viáveis/número de fêmeas vivas. $\mathrm{O}$ experimento foi conduzido a $22^{\circ} \mathrm{C} \pm 1^{\circ}, \mathrm{UR}$ de $75 \% \pm 10 \mathrm{e}$ fotofase de $12 \mathrm{~h}$. Os produtos foram classificados quanto à toxicidade de acordo com IOBC/WPRS, onde: classe $1=\mathrm{E}<30 \%$ (neutro); classe $2=30 \%<\mathrm{E}<79 \%$ (levemente nocivo); classe 3 $=80 \%<\mathrm{E}<99 \%$ (moderadamente nocivo) e classe $4=\mathrm{E}>99 \%$ (nocivo) (Hassan et al., 1985; Bakker et al., 1992; Reis et al., 1998)).

TABELA 1 - Inseticidas testados sobre Neoseiulus californicus

\begin{tabular}{|c|c|c|c|c|c|}
\hline Produto comercial & Nome técnico & $\begin{array}{l}\text { Formulação } \\
\text { concentração }\end{array}$ & $\begin{array}{c}\text { Classe } \\
\text { toxicológica }\end{array}$ & $\begin{array}{c}\text { Grupo } \\
\text { químico }\end{array}$ & $\begin{array}{c}\text { Praga }^{1} \\
\text { homologada }\end{array}$ \\
\hline Decis & Deltametrina & CE 25 & II & Pire tró ide & A \\
\hline Dimeto a to & Dimethoate & $\mathrm{CE} 400$ & I & Organofosforado & $\mathrm{E} ; \mathrm{A} ; \mathrm{G}$ \\
\hline Folidol & Parathion methyl & CE 600 & I & Organo fosforado & $\mathrm{E} ; \mathrm{Q} ; \mathrm{G} ; \mathrm{S}$ \\
\hline Gusathion & Azinphos ethyl & $\mathrm{CE} 400$ & I & Organo fosforado & E \\
\hline Imidan & Phosmet & PM 500 & II & Organofosforado & $\mathrm{G} ; \mathrm{A}$ \\
\hline Lebaycid & Fenthion & CE 500 & II & Organofosforado & $\mathrm{E} ; \mathrm{G} ; \mathrm{A}$ \\
\hline Malatol & Malathion & CE 500 & III & Organofosforado & $\mathrm{E} ; \mathrm{Q} ; \mathrm{S}$ \\
\hline Sumithion & Fenitrothion & CE 500 & II & Organo fosforado & $\mathrm{E} ; \mathrm{G} ; \mathrm{A} ; \mathrm{S}$ \\
\hline Triclorfon & Triclorfon & PS 800 & II & Organofosforado & $\mathrm{G}$ \\
\hline
\end{tabular}

TABELA 2 - Toxicidade de inseticidas a fêmeas adultas de Neoseiulus californicus expostas aos mesmos por 96 horas em condições de laboratório, originadas de macieira com controle biológico, Agropastoril Rincão das Flores, Vacaria-RS.

\begin{tabular}{|c|c|c|c|c|c|c|c|}
\hline Nome técnico ${ }^{1}$ & $\begin{array}{c}\text { Concentração } \\
(\mathrm{ppm})\end{array}$ & $(\mathrm{Mc})^{3}(\%)$ & $\begin{array}{c}\text { Sobrevivente } \\
100 \%-\mathrm{Mc}\end{array}$ & $\mathrm{R}^{4}$ & $\mathrm{E}_{\mathrm{r}}^{5}$ & $E^{6}(\%)$ & Classes $^{7}$ \\
\hline Azinphos ethy & 1500 & 100,0 & 0,0 & 0,0 & 0,0 & 100,0 & 4 \\
\hline Deltametrina & 400 & 85,7 & 14,3 & 0,0 & 0,0 & 100,0 & 4 \\
\hline Dimethoate & 1500 & 57,1 & 42,9 & 3,2 & 0,7 & 68,4 & 2 \\
\hline Fenitrotion & 2000 & 64,3 & 35,7 & 4,0 & 0,9 & 66,8 & 2 \\
\hline Fenthion & 1000 & 100,0 & 0,0 & 0,0 & 0,0 & 100,0 & 4 \\
\hline Malathion & 1500 & 28,6 & 71,4 & 5,7 & 1,3 & 5,3 & 1 \\
\hline Parathion methyl & 1000 & 71,4 & 28,6 & 6,3 & 1,5 & 58,5 & 2 \\
\hline Phosmet & 2000 & 35,7 & 64,3 & 2,8 & 0,6 & 58,5 & 2 \\
\hline Triclorfon & 2000 & 50,0 & 50,0 & 4,3 & 1,3 & 36,9 & 2 \\
\hline
\end{tabular}

1 Nomenclatura segundo Andrei (1990)

2 Concentração de produto comercial

3 Mortalidade corrigida - Mc (Abbott, 1925)

4 Produção média de ovos por fêmea - R 


\section{RESULTADOS E DISCUSSÃO}

Os resultados dos efeitos dos inseticidas sobre $N$. californicus expostos aos mesmos por 96 horas, expressos em mortalidade corrigida $(\mathrm{Mc})$, percentagem de sobrevivência (100$\mathrm{Mc})$, efeito reprodutivo $\left(\mathrm{E}_{\mathrm{r}}\right)$, efeito total $(\mathrm{E} \%)$ e classe toxicológica, são apresentados na Tabela 2 .

Os agroquímicos azinphos ethyl, deltametrina e fenthion mostraram-se nocivos, causando $100 \%$ da mortalidade de fêmeas em 24 horas após a exposição aos mesmos, demonstrando forte efeito de choque, enquanto dimethoato, fenitrotion, parathion methyl, phosmet e triclorfon apresentaram um efeito de choque menor, o que possibilitou oviposição pelas fêmeas viáveis durante todos os dias de observação.

$\mathrm{O}$ efeito dos inseticidas na reprodução $\left(\mathrm{E}_{\mathrm{r}}\right)$ de fêmeas sobreviventes de $N$. californicus nos tratamentos malathion, parathion e triclorfon produziram proporcionalmente mais ovos do que a testemunha, apresentando valores de $\mathrm{E}_{\mathrm{r}}$ maiores do que 1 (Tabela 2). O cálculo de $\mathrm{E}_{\mathrm{r}}$ levou em consideração a Mc e a produção média de ovos por fêmeas sobreviventes, neste caso, mesmo que o efeito negativo do inseticida sobre a população seja elevado, as fêmeas sobreviventes mantiveram as suas características genéticas e o seus potenciais de ovoposição. Assim, embora a mortalidade no tratamento malathion tenha sido superior à testemunha, respectivamente $33 \%$ e $7 \%$, o efeito sobre a reprodução foi favorável às fêmeas sobreviventes do tratamento malathion em função de estas terem produzido 97 ovos contra 95 ovos na testemunha.

Esse elevado valor de $\mathrm{E}_{\mathrm{r}}$ pode estar relacionado com os tratamentos de inseticidas fosforados realizados no pomar entre a liberação e a coleta dos adultos de $N$. californicus, pois é possível que tenha havido seleção destas populações, tornandoas mais tolerantes a estes agroquímicos. A seleção de populações resistentes devido à intensidade dos tratamentos fitossanitários foi observada por diferentes autores (Meyer, 1974; Caccia et al., 1985; Corino et al., 1986).

Os resultados obtidos sugerem-nos que os produtos da classe 4 não devam ser utilizados em programas de controle biológico, pois o alto grau de mortalidade apresentado em laboratório deverá ser reproduzido a campo (Hoy \& Conley, 1987). Entretanto, os ácaros predadores poderão desenvolver uma outra estratégia de seletividade que não seja a fisiológica, necessitando de que os testes de seletividade sejam repetidos a campo (Reis et al., 1998).

No caso de fenthion e de phosmet, os resultados de laboratório confirmam os efeitos obtidos no campo por Monteiro (1993), que mostrou que a população de $N$. californicus foi reduzida após pulverizações com fenthion, enquanto phosmet não apresentou o mesmo efeito e não influenciou o equilíbrio entre as populações dos ácaros vermelho e predador.

Os resultados encontrados neste trabalho não podem ser extrapolados para outras regiões de macieiras, pois estes são dependentes da espécie, do microambiente e da seleção da população exercida pelos tratamentos fitossanitários.

\section{AGRADECIMENTOS}

Ao Ilmo. Sr. Leandro Kolodny, diretor da Agropastoril Rincão das Flores Ltda., pelo financiamento deste projeto. Ao Dr. José Fernando da S. Protas (CNPUV) e Dr. Gilberto de Moraes (ESALQ-USP), pelo apoio à execução deste trabalho.

\section{REFERÊNCIAS BIBLIOGRÁFICAS}

ABBOTT, W. S. A method of computing the effectiveness of na insecticide. Journal of Economic Entomology, Maryland, v. 18, p. 265-267, 1925 .

ANDREI, E. Compêndio de defensivos agrícolas. 3. ed. São Paulo: Andrei (Ed.), 1990. 478p.

A.N.P.P. Les actions secondaires des produits phytosanitaires. Paris: Association Nationale pour la Protection des Plantes, 1987. 4: 60p.

BAKKER, F. M.; GROVE A.; BLÜMEL S.; CALIS J.; OOMEN P. Side-effect test for phytoseiids and their rearing methods. IOBC/ WPRS Bulletin, Montfavet, v. 15, p. 61-81, 1992.

BESSON, J.; JOLY E.; TOUZEAU J. Les actions secondaires des antiparasitaires agricoles. Phytoma, Paris, v. 296, p. 18-26, 1978.

BLEICHER, J.; GASSEN D. N.; RIBEIRO L. G.; TANAKA H.; ORTH A. I. A mosca-das-frutas em macieira e pessegueiro. Florianópolis: Empresa Catarinense de Pesquisa Agropecuária, 1982. (Boletim Técnico, 19) 28p.

CACCIA R.; BAILLOD M.; GUIGNARD E.; KREITER S. Introduction d'une souche de Amblyseius andersoni Chant (Acari, Phytoseiidae) résistant à l'azinphos, dans la lutte contre les acariens phytophages en viticulture. Revue suisse Viticulture, Arboriculture et Horticulture, Lausanne, v. 17, p. 285-290, 1985.

CORINO L.; BAILLOD M.; DUVERNEY C. Resistenza di Kampimodromus aberrans (Oudemans) al parathion e lotte biologica control gli acari fitofagi in viticoltura. Vidnevini, Roma, v. 4, p. 39-42, 1986.

FAUVEL, G.; ATGER P. Etude de l'évolution des insectes auxiliaires et de leurs relation avec le psille du poirier (Psylla pyri L.) et l'acarien rouge (Panonychus ulmi Koch) dans deux vergers du Sud-Est de la France en 1979. Agronomie, Paris, v. 1, n. 9, p. 813-820, 1981.

HASSAN, S. A.; BIGLER F.; BLAISINGER P.; BOGENSHÜTZ H.; BRUN J.; CHIVERTON P.; DICKLER E.; EASTERBROOKM. A.; EDWARDS P.J.; ENGLERT W. D.; FIRTH S. I.; HUANG P.; INGLESFIELDD.; KLINGAUF F.; KÜHNER C.; OVERMEER W. P. J.; PLEVOETS P.; REBOULET J. N.; RIECKMANN W.; SAMSOE-PETERSEN L.; SHIRES S. W.; STÄUBLI A.; 
STEVENSON J.; TUSET J. J.; VANWETSWINKEL G.; VANZON Q. Standard methods to test the side-effects of pesticides on natural enemies of insects and mites developed by the IOBC/ WPRS working group "Pesticides and Beneficial Organisms". EPPO Bulletin, Oxford, v. 15, p. 214-255, 1985.

HOY, M. A.; CONLEY J. Toxicity of pesticides to western predatory mite. California Agriculture, Oakland, v. 41, p. 12-14, 1987.

MONTEIRO, L.B. Etude de la relation entre l'alimentation hydrique, la physiologie du pommier et le développement de l'araignée rouge Panonychus ulmi (Koch, 1836) (Acari: Tetranychidae). 1991. 141f. Tese (Doutorado em Ciências) - Ecole Nationale Supérieure Agronomique de Montpellier, Montpellier, 1991.

MONTEIRO, L. B. Controle biologique de Panonychus ulmi (Koch) (Acari: Tetranychidae) en fonction du programme de traitement contre la mouche des fruits dans la région de Vacaria (Brésil). In. CONFÉRENCE INTERNATIONALE RAVAGERS EN AGRICULTURE, 3., 1993, Montpellier. Anais... v. 2, p. 611-619.

MONTEIRO, L. B. Manejo Integrado de Panonychus ulmi em macieira. Primeiras experiências com a introdução de Neoseiulus californicus. Revista Brasileira de Fruticultura, Cruz das Almas, v. 16, n.1, p.46-53, 1994.

MORAES, G. J.; MCMURTRY J. A.; DENMARK H. A A catalog of the mite family Phytoseidae. Brasília: EMBRAPA-DDT, 1986. $353 \mathrm{p}$.

MCMURTRY, J. A.; HUFFAKER C. B.; VAN DE VRIE V.. Ecology of tetranychid mites and their natural enemies: A review. I. Tetranychid enemies: their biological characters and the impact os spray practices. Hilgardia, Berkeley, v. 40, n. 11, p. 331-390, 1970.

MEYER R. H. Management of phytophagous and predatory mites in Illinois orchards. Environmental Entomology, Steeles Tavern. v. 3, p. 333-340, 1974.

OVERMEER, W. P. J. Toxicological methods. In: Helle \& Sabelis, Spider mites their biology, natural enemies and control. Amsterdan, New York: Elvesier, 1985, 1B, p. 183-189.

REIS, P. R.; CHIAVEGATO, L. G.; MORAES, G. J.; ALVES E. B.; SOUZA, E. O. Seletividade de agroquímicos ao ácaro predador Iphiseiodes zuluagai Denmark \& Muma (Acari: Phytoseiidae). Anais da Sociedade Entomológica do Brasil, Piracicaba, v. 27, n. 2,p. 265-274, 1998.

SALLES, L. A. B.; KOVALESKI A. Mosca-das-frutas em macieira e pessegueiro no Rio Grande do Sul. Hort Sul, Pelotas, v. 1, p. 5 9, 1990a

SALLES, L. A. B.; KOVALESKI A. Inseticidas para o controle da mosca-das-frutas. Hort Sul, Pelotas, v. 1, p. 10-11, 1990 b.

SALLES, L. A. B. Mosca-das-frutas (Anastrepha spp.): Bioecologia e controle. Pelotas: EMBRAPA-CNPFT, 1991. 16p. (Documento, 41)

SPV. Les actions secondaires des produits phytosanitaires. 5. ed. Paris: Service de la Protection des Végétaux, 1983. 96p. 